\title{
Wallenstein - Selbsttäuschung und Identitätsbrüche im Spannungsfeld der Politik
}

Wallenstein - auto-aveuglement et fractures identitaires dans le champ de forces contradictoires de la politique

Wallenstein - self-blinding and identity fractures in the contradictory force field of politics

\section{Gert Sautermeister}

\section{OpenEdition}

Journals

Édition électronique

URL : http://journals.openedition.org/ceg/1758

DOI : $10.4000 /$ ceg. 1758

ISSN : 2605-8359

\section{Éditeur}

Presses Universitaires de Provence

Édition imprimée

Date de publication : 1 décembre 2014

Pagination : 137-155

ISSN : 0751-4239

Référence électronique

Gert Sautermeister, «Wallenstein - Selbsttäuschung und Identitätsbrüche im Spannungsfeld der Politik », Cahiers d'Études Germaniques [Online], 67 | 2014, Online erschienen am: 17 Dezember 2017, abgerufen am 07 November 2020. URL : http://journals.openedition.org/ceg/1758 ; DOI : https:// doi.org/10.4000/ceg. 1758 


\title{
Wallenstein - Selbsttäuschung und Identitätsbrüche im Spannungsfeld der Politik
}

\author{
Gert SAUTERMEISTER \\ Universität Bremen
}

\begin{abstract}
Die folgende Analyse der Wallenstein-Gestalt ${ }^{1}$ versteht unter Selbsttäuschung eine zwischen den Polen Wahrheit und Lüge mehrdeutig schillernde Haltung. Charakteristisch für sie ist ein Bewusstseinsmangel, aufgrund dessen das Subjekt die Wahrheit über sich selbst verkennt, ohne deshalb zu lügen - denn Lügen setzt eine Absichtlichkeit voraus, die der Selbsttäuschung fehlt. Sie lässt sich vielmehr als eine unbewusste Selbstverkennung bezeichnen, bei der das Subjekt die Konsequenzen seines Tuns und Lassens vor dem eigenen Bewusstsein verhüllt. Wallenstein macht zwar aus seinen Gedanken, Affekten und Stimmungen kein Geheimnis, aber er scheut sich davor, ihre Folgen zu bedenken. Er spielt mit Möglichkeiten, ergeht sich in Träumen - bis letztere ihn überspielen. Er dreht und wendet seine Identität nach vielen Richtungen, probiert verschiedene Entwürfe aus, ohne sich zu fragen, ob er sie miteinander und mit sich selbst vereinbaren kann. So gerät er in den Raum der Selbsttäuschung. Im Privatleben mag die Selbsttäuschung unerfreuliche Kreise ziehen und einen in Schuld verstricken, vielleicht auch ohne schwerwiegende Folgen zerrinnen; im Feld der Politik und der Macht kann sie ein Verhängnis von überpersönlicher geschichtlicher Tragweite zeitigen. Das demonstriert Schillers Wallenstein, eines der bedeutendsten deutschen Geschichtsdramen ${ }^{2}$, nicht zuletzt dank seiner scharfsichtigen Psychologie der Selbsttäuschung. Ihr ist noch wenig Aufmerksamkeit zuteil geworden, umso lohnender erscheint der Versuch
\end{abstract}

\footnotetext{
${ }^{1}$ Mein Versuch ist die Umarbeitung eines Aufsatzes, der an entlegener Stelle erschienen ist: Gert SAUTERMEISTER, „Das Spannungsverhältnis von Politik und Moral. Zur Modernität der Gestalt Wallensteins“, in Friedrich v. Schiller zum 200. Todestag, hrsg. v. Ortsvereinigung Hamburg der Goethe-Gesellschaft in Weimar (E.V. Jahresgabe 2006), S. 55-84.

${ }^{2}$ Über die bewundernswerte „Architektur der Trilogie" unterrichtet differenziert PeterAndré ALT, Schiller. Leben - Werk - Zeit, München, C. H. Beck, 2004, 2. Bd., S. 428-438.
} 
einer Analyse. Sie könnte der Wallenstein-Figur, die ihrer umstrittenen Vieldeutigkeit wegen Schiller besonders fesselte, neue Seiten abgewinnen. ${ }^{3}$

Wallensteins Selbsttäuschung beruht auf heterogenen Antriebskräften, auf einer Vielzahl undurchschauter Widersprüche, die der geschichtliche Prozess miterzeugt hat. Diese geschichtlich-politische Bedingtheit eines dissoziierten Selbst verweist auf ein für die deutsche Klassik ungewöhnliches Menschenbild, von dem die Forschung noch kaum Kenntnis genommen hat. ${ }^{4}$ Goethe hatte die Entwicklung der Person als eine organische aufgefasst: aus einem Wesenskern entfaltet sie harmonisch die ihr eingeborenen Anlagen und führt sie zur Synthese. Gegenüber diesem Prozess der Integration menschlicher Grundkräfte mutet die Desintegration persönlicher und historisch veränderlicher Wesenszüge im Wallenstein modern an. Im Kräftefeld der Politik und Moral vereinfacht jedoch der Protagonist seine widerspruchsvolle Identität radikal. Er isoliert einen Kern dieser Identität als sein Lebensgesetz von den ethischen Prämissen politischen Handelns. Gegen diese Halbwahrheit einer geschichtlichen Existenz behauptet Max Piccolomini im Namen der Moral eine bedingungslose ,tödliche' Wahrheit. Im Medium seines Todes erkennt Wallenstein am Ende den Substanzverlust seines eigenen Lebens.

\section{Wallensteins Trauma. Seine heterogenen Projekte}

Ich rolle mein Thema von einem finalen Stadium der Selbsttäuschung auf, als Wallenstein sich erstmals mit ihr konfrontiert sieht und versuche dann ihre

${ }^{3} \mathrm{Zu}$ Schillers Annäherungen an den historischen Wallenstein und damit an seine Quellen für das Drama vgl. Norbert OELLERS, „Wallenstein“, in Schiller-Handbuch. Leben - WerkWirkung, hrsg. v. Matthias LUSERKE-JAQUI unter Mitarbeit von Grit DOMMES, Stuttgart/ Weimar, Metzler, 2005, S. 126ff.

${ }^{4}$ Eine Haupttendenz der Forschung zum Wallenstein zielt darauf, den Protagonisten als Charakter mit feststehenden Eigenschaften zu erfassen. So auch die bedeutenden Monographien von Peter-André Alt (Anm. 2) und von Dieter BORCHMEYER, Macht und Melancholie. Schillers Wallenstein, Neckargemünd/ Wien, Mnemosyne, 2003, überarbeitete Neuauflage des 1988 im Athenäum Verlag erschienenen Buches. Borchmeyer deutet Wallenstein als melancholischen Charakter mit vielfältigen aufschlussreichen Eigenschaften, während Alt ihn als ,bedenkenlosen Machtmenschen“ mit „düster-abweisenden Charakterzügen“ darstellt (S. 441), dem überpersönliche Zielsetzungen fremd sind; eine „Kette der persönlichen Irrtümer“ (S. 445), die mit seinem Sternenglauben „verbunden“ sind (S. 446), führen diesen ,pragmatischen“ Materialisten (vgl. S. 440f.) und an sich klugen Strategen „Graciánscher Prägung“ (S. 442) in die Katastrophe. Unbeschadet etlicher treffender Charakteristika im einzelnen vermisse ich an den Darstellungen der Gestalt Wallensteins den Nachweis der verschiedenen Stadien, die sie durchläuft, also ihre im geschichtlich-politischen Prozess eintretenden Veränderungen. Einen Wandel in der Gestalt des Feldherrn stellt dagegen Walter MüLLER-SEIDEL in Rechnung: Friedrich Schiller und die Politik. „Nicht das Große, nur das Menschliche geschehe“, München, C. H. Beck, 2009, vgl. S. 122-146. 
Anfänge und Steigerungen zu erfassen. Zu Beginn von „Wallensteins Tod“朕 dem erwähnten finalen Stadium - besichtigt Wallenstein mit erregter Hoffnung die eben eingetroffene, glückverheißende Konstellation der Gestirne. $\mathrm{Zu} \mathrm{Seni,} \mathrm{seinem} \mathrm{Astrologen,} \mathrm{gewandt,} \mathrm{bemerkt} \mathrm{er} \mathrm{triumphierend,}$ dass seine „Segenssterne, Jupiter und Venus“, endlich „den tückschen Mars in ihre Mitte" nehmen und ihn, ,,den alten Schadensstifter“, zwingen, ,mir zu dienen“. (Tod, V. 11-14). ,Jetzt muß / Gehandelt werden, schleunig“, entfährt es Wallenstein (Tod, V. 32f.), der sein Handeln lange aufgeschoben hat. Just in diesem Augenblick - Schillers dramatische Ironie fährt ernüchternd in den „glückseligen Aspekt“ (Tod, V. 9) - unterbrechen Wallensteins Vertraute die Sternenschau mit der Nachricht, dass der Unterhändler Sesin, ein Verbindungsmann Wallensteins, der an seiner Stelle geheime Verhandlungen mit den Reichsfeinden, den Schweden und Sachsen, führt, gefangen genommen wurde und dem Kaiser am Wiener Hof, dem Reichsoberhaupt und Kriegsherrn, überstellt wird. Der Hof wird Sesin das Geständnis über Wallensteins Verhandlungen mit den Feinden des Reichs abpressen, was die schimpfliche Absetzung des Feldherrn als eines „Landesverräters“ zur Folge hätte (Tod, V. 107). Der Gedanke ist für Wallenstein unerträglich, hat er doch einen Verrat nie verbindlich beschlossen und Verhandlungen mit den Reichsfeinden nur zum Schein geführt, um diese für seine persönlichen Zwecke auszunützen und schließlich an die Wand zu spielen. „Ein böser, böser Zufall -“, so kommentiert er den Umschwung der Dinge (Tod, V. 98).

Mit diesem Zufall ist Wallensteins Sternenschau und Sternenglaube als Täuschung entlarvt, als eine Selbsttäuschung des Helden, die umso verhängnisvoller ist, als Wallenstein unter Berufung auf die ausbleibende günstige Sternenkonstellation ein gezieltes Handeln bisher aufgeschoben hat. Er, das Musterbild des raschen entschlossenen Tathelden, hat über längere Zeitstrecken hinweg sich als Zögerer und Zauderer aufgeführt, zum Verdruss seiner Vertrauten und Verwandten. Einer von ihnen, Feldmarschall Illo, ist seines Zögerns längst überdrüssig und verweist ihn auf die Konstellation nicht der Gestirne, sondern der historisch-politischen Gegebenheiten, die das Handeln zum dringenden Gebot machen: „O du wirst auf die Sternenstunde warten,/ Bis dir die irdische entflieht! Glaub mir,/ In deiner Brust sind deines Schicksals Sterne./ Vertrauen zu dir selbst, Entschlossenheit/ Ist deine Venus!“ (Picc., V. 960ff.)

Nun ist in der Tat dem Sternenseher und Zögerer die „,irdische“ Stunde entflohen. Was nötigte Wallenstein zu seinem astrologischen Glauben, dieser Selbsttäuschung, die sich als Verhängnis herausstellt? Das Drama liefert zur Lösung der Frage einige Fingerzeige, die auf frühere Erfahrungen des Feldherrn zurückführen, namentlich auf den sogenannten Regensburger Fürstentag. Wallenstein hatte „vor Jahren“ Erstaunliches zustande gebracht,

\footnotetext{
${ }^{5}$ Zitiert werden Die Piccolomini und Wallensteins Tod nach Friedrich SCHILLER, Sämtliche Werke, Bd. 2, Dramen II, hrsg. von Gerhard FRICKE und Herbert G. GOEPFERT in Verbindung mit Herbert STUBEnRAUCH, München, Carl Hanser, 1974.
} 
hatte „ein Heer wie aus dem Nichts hervorgerufen“ (Tod, V. 289f.), hatte den Kaiser aus höchster Bedrängnis befreit, indem er die „Landeshoheit“ der Fürsten niedergetreten, die rechtmäßigen „Ordnungen des Reichs“ rücksichtslos verletzt und der „Stärke fürchterliches Recht“" geübt hatte (Tod, V. 606ff.) - sehr zum Gefallen des Kaisers, der „diesen Freveltaten/ Sein kaiserliches Siegel“ aufdrückte (Tod, V. 613f.). Den Dank, genauer: den Undank für seine Dienste stattete ihm der Kaiser auf dem Regensburger Fürstentag ab, als er seinen Feldherrn den Klagen der Landesfürsten preisgab und ihn seines Postens enthob. Es war ein Akt politischer Unmoral mit traumatischen Folgen für Wallensteins Psyche und seine politische Mentalität. Erst als der Kaiser Jahre später erneut in „herbe Not“ geriet, berief er Wallenstein wieder zum obersten Befehlshaber (Tod, vgl. V. 570ff.). Doch diese Berufung konnte das Trauma nicht auflösen, das Wallenstein seit dem Fürstentag verfolgt. Es ist das Trauma des Argwohns gegenüber der Loyalität des Kaisers, des Bedürfnisses nach Rache für die erlittene Schmach und der Furcht vor einer neuen Absetzung. Wallensteins Gattin kommentiert im Dialog mit ihrer Tochter das veränderte Seelenleben Wallensteins wie folgt:

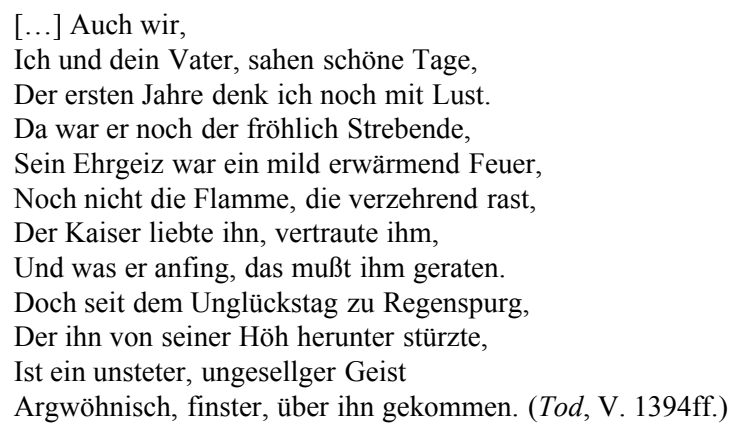

Die Auskunft der Herzogin verdeutlicht ein Grundelement der dramatischen Figurengestaltung Schillers - sein Verständnis der menschlichen Psyche als eines dynamischen Kräftefelds, das geschichtlichem Wandel unterworfen ist. Der Herzogin zufolge hat das Trauma des Regensburger Fürstentags eine neue Seite in Wallensteins geistig-seelischer Existenz hervorgebracht - seinen vielzitierten Sternenglauben:

Ihn floh die Ruhe, und dem alten Glück,

Der eignen Kraft nicht fröhlich mehr vertrauend

Wandt er sein Herz den dunklen Künsten zu,

Die keinen, der sie pflegte, noch beglückt. (Tod, V. 1406f.)

Wallenstein teilt zu dem Zeitpunkt, da die Herzogin diese Aussage macht, ihre Skepsis keineswegs. Ihm scheint seine Erkundung der Gestirne vielmehr die Erkenntnis der „Tiefen der Natur“ und der „Geisterleiter“ zu verbürgen, die, so glaubt er, ,aus dieser Welt des Staubes/ Bis in die Sternenwelt $[\ldots] /$ Hinauf sich baut". (Picc., V. 977ff.) Es ist eine kosmische Wesensschau, die 
Wallenstein anstrebt, eine Schau mit platonischem und mystischem Einschlag, die auf das Erkennen einer ,zentralischen Sonne“ ausgerichtet ist, eines Urbilds, das nur einem im Sternzeichen des Jupiter Geborenen zugänglich ist, einem der „hellgebornen, heitern Joviskinder“ (vgl. Tod, V. 983ff.). Wallensteins Enthusiasmus an dieser Stelle ist der eines Auserwählten, eines Aristokraten unter der Masse all derjenigen, die unter anderen, trivialen Sternzeichen geboren wurden, im Zeichen des „bleichen, bleifarbnen“ Saturn beispielsweise (Tod, V. 971f.). Schiller verleiht der Gestalt seines Helden eine neue, interessante Facette, er gesellt der vita activa Wallensteins die vita contemplativa zu, und es lässt sich unschwer erkennen, dass Wallenstein an dieser Stelle durch die Kraft der Phantasie sich entschädigt für die im politisch-geschichtlichen Prozess erlittene Demütigung. Er adelt seine Person und überwölbt seine Schmach durch die "heitere“ Wesensschau eines vom Jupiter-Gestirn ausgezeichneten Metaphysikers. So führt er beispielhaft die Selbstbehauptung eines Menschen vor, dessen Selbstbewusstsein gekränkt wurde, führt sie vor durch die Entfaltung seiner kompensatorischen Phantasie. Es sind hundert andere Möglichkeiten der Selbstbehauptung in existentiellen Krisensituationen denkbar - wesentlich ist hier die Einsicht, dass es dem Menschen gemäß ist, sich einer Lebenskrise durch ein verändertes Selbstverständnis und eine neue Sinngebung zu erwehren.

Doch damit begnügt sich Wallenstein keineswegs. Er möchte aus der kosmischen Sternenschau auch einen politisch pragmatischen Nutzen ziehen. Mittels der Gestirne will er eine gezielte Strategie gegen die Wechselfälle in der geschichtlichen Welt entwickeln. Das Verhängnis, das ihn einmal betroffen hat, soll ihn kein zweites Mal heimsuchen. Überraschungen, missliebige Zufälle, Schicksalsschläge - all das soll künftig ausgeschaltet werden. Die „Gestirne“, so glaubt er, bezeichnen dem politisch Handelnden den Zeitpunkt, an dem er zur Tat schreiten darf, zu einer erfolgreichen und von Glück gesegneten Tat. Auf die entsprechende Sternenkonstellation wartet Wallenstein. Sie ist es, die sein Zögern bedingt. ${ }^{6}$

Das mag manchem befremdlich scheinen, mag ein Lächeln hervorrufen. Man bedenke jedoch, dass hier ein zutiefst Verunsicherter am Werk ist, der eine verlässliche Orientierung sucht, eine sichere Prognose. Und man bedenke ferner, dass Prognosen als sinngebende Orientierungsmarken bis heute nichts Ungewöhnliches sind. In der Epoche Schillers stellte der Prediger und Philosoph Lavater anhand von physiognomischen Merkmalen Prognosen über den Charakter der Menschen auf. Ganze Generationen nahmen seine fahrlässigen Schlüsse ernst und vertrauten ihnen blind, von der Hoffnung angestachelt, sie könnten so Wegweiser für ihre Menschenkenntnis erhalten. Heute erlassen noch immer Sterndeuter Prognosen für die

\footnotetext{
${ }^{6}$ Unter diesem (textadäquaten) Aspekt nimmt sich Safranskis These, Wallenstein mache ,in keinem Fall [...] seine Entscheidungen von der Sternenkonstellation abhängig“, als Fehlurteil aus. Rüdiger SAFRANSKI, Friedrich Schiller oder die Erfindung des deutschen Idealismus, München, Carl Hanser, 2004, S. 460.
} 
unmittelbar bevorstehende Zukunft, prognostizieren fünf Wirtschaftsweise ökonomische Entwicklungen, die sie im Handumdrehen zu korrigieren pflegen, prophezeien Fachleute die nächsten Phasen auf den Aktienmärkten, die nur selten eintreffen, errechnen Kultusministerien den zukünftigen Bedarf von Lehrerstellen, die nie oder nur teilweise geschaffen werden. Durch den Weltlauf kursiert eine Flut von Voraussagen, auf die man seine Sicherheit glaubt gründen zu können. Die Irrationalität des Lebens und der Geschichte soll durch Rationalität, durch Vorausberechnungen kalkulierbar werden. Wallensteins Sternendeutung ist dafür nur ein besonders auffälliges Beispiel, erwachsen aus einer traumatischen Verunsicherung. Schiller, so darf man folgern, sieht im politisch Handelnden keineswegs nur einen Repräsentanten von Sachzwängen, eine heute sehr verbreitete Unterstellung. Sie unterschlägt, dass in politisches Tun stets auch die persönlichen Affekte und biographischen Erfahrungen der Akteure hineinwirken können - bis zur Selbsttäuschung. Eben davon weiß der Dramatiker Schiller Eindringliches zu erzählen.

Dem Regensburger Fürstentag entstammt aber auch ein sublimierter Rachegedanke Wallensteins, den er in ein politisches Gewand kleidet - seine neue Reichsidee. Als zu Beginn der Piccolomini ein Kriegsrat des Kaisers, von Questenberg mit Namen, in Wallensteins Feldlager auftaucht und ihm kaiserliche Befehle überbringt, weist der ihn, eingedenk der Regensburger Schmach, in die Schranken. Er, der für den Kaiser auf Kosten der Reichsfürsten zu Felde gezogen war, hat inzwischen einen neuen Kurs eingeschlagen:

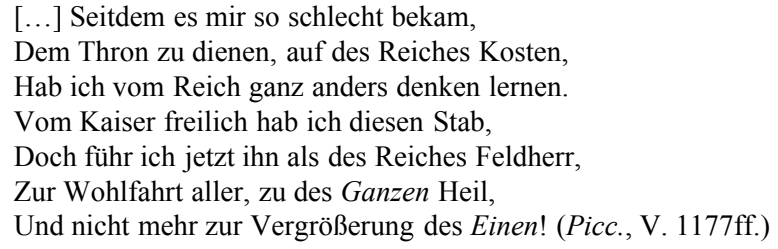

Wallenstein riskiert mit dieser offenen Rede viel. Er stellt die allgemeinen Interessen des Reichs provokativ über die besonderen des Kaisers und scheut nicht länger die Konfrontation mit dem Wiener Hof. Indem Schiller ihn - vor seinen Generälen - zum Sprecher für die „Wohlfahrt aller“, für „des Ganzen Heil" macht, knüpft er an eine seiner bevorzugten Gestaltungstendenzen an: er weist der Heldenrolle die Verantwortung für das allgemeine Beste zu. Insofern scheint Wallenstein das Regensburger Trauma glücklich aufzulösen und sein persönliches Bedürfnis nach Rache am Kaiser in einer überindividuellen politischen Idee aufheben zu können. „Mich soll das Reich als seinen Schirmer ehren," lässt er einen seiner engsten Vertrauten wissen: „Reichsfürstlich mich erweisend, will ich würdig/ Mich bei des Reiches Fürsten niedersetzen.“ (Picc., V. 835ff.) 
Insgeheim zehrt an dieser schönen Utopie des Ideenpolitikers jedoch ein egozentrisches Interesse des Machtpolitikers Wallenstein. Wiederholt führt Schiller, wie wir wissen, in seiner Dramatik den Widerstreit von Idee und Macht vor, aber nirgendwo hat er ihn so hintergründig und so vieldeutig ausgearbeitet wie im Wallenstein. Der Held und Kriegsfürst will seine Hausmacht erweitern und begehrt nicht mehr und nicht weniger als eine Königskrone für seine aufwendigen militärischen Mühen. Seiner Tochter will er eines Tages „den Kranz des kriegerischen Lebens [...] in einen königlichen Schmuck verwandelt“" um die „schöne Stirne flechten“ (Picc., V. 748ff.).

Der „königliche Schmuck“ soll die böhmische Krone sein, auf die das Machtverlangen Wallensteins zielt. Sein Traum ist es, als des Reiches Schirmherr zugleich König von Böhmen zu sein, und dergestalt das Allgemeine mit seinen besonderen Interessen zu verschränken. Des Kaisers besondere Interessen dagegen zielen auf die Erhaltung Böhmens unter seinem Szepter, seiner Regierungsgewalt, die durchaus auch die Gewalt gegen Böhmens protestantische Majorität einschließt. Octavio Piccolomini, der vermeintliche Freund Wallensteins, der sich als sein Intimfeind enthüllen wird, bringt den Konflikt prägnant auf den Punkt, wenn er seinem Sohn Max die Absichten Wallensteins deutet:

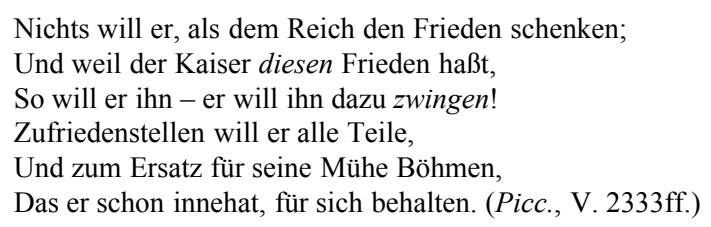

Das zentrale Problem, das sich hiermit Wallenstein stellt, lautet: Wie kann er in den Besitz der Krone Böhmens gelangen? An diesem heiklen Punkt setzt nun Schiller ein Machtspiel in Szene, das seinesgleichen sucht in der Geschichte des deutschen Dramas.

Schiller hebt am Beispiel seines Helden einen Grundzug der Macht hervor: ihre Verführungskraft. Es liegt in der Dynamik der Macht, dass sie ihren Träger in die Versuchung zu größerer Machtfülle führt. Dass sie sich seiner Egozentrik andient und seinem Narzissmus schmeichelt. Und dass sie ihn zum Spieler macht. Davon erzählt Wallensteins Monolog - diese grandiose Selbstdurchleuchtung eines Liebhabers, eines Träumers und eines Beischläfers der Macht (Tod, I, 4). Er habe sich, bekennt Wallenstein, bloß „,in dem Gedanken“ der Macht gespiegelt, habe nur ,an dem Gaukelbilde [...] der königlichen Hoffnung“ sich ergötzt, und doch auch „,das Herz genährt mit diesem Traum“. Es sei ein unwiderstehlicher Reiz gewesen, die „Freiheit“ zum Spiel mit der Macht auszukosten, einem zum Abenteurertum verlockenden Spiel. Das wird schon vor Wallensteins Monolog deutlich. Aus den verschiedenen Äußerungen des Feldherrn gegenüber seinen Vertrauten, Illo und Terzky, wird der Abenteurer Wallenstein als Maskenspieler kenntlich, der sich die Reichsfeinde, Schweden und Sachsen, mit dem Ziel 
der Machterweiterung zunutze machen will, um sie schließlich aus dem Land zu jagen: „Es soll im Reiche keine fremde Macht/ Mir Wurzel fassen“ (Picc., V. 838f.) - Die potentiellen Bündnispartner durchschauen jedoch Wallensteins Täuschungsmanöver nach und nach und drohen die fruchtlosen Verhandlungen mit seinen Gefährten abzubrechen. Entnervt halten Illo und Terzky dem Feldherrn seine „krummen Wege“ und wechselnden „Masken“ vor (Picc., V. 847ff.) und drängen auf einen entschiedenen Seitenwechsel zugunsten der Schweden, worauf Wallenstein seine übergeordnete Reichsidee ins Feld führt: „Mich soll das Reich als seinen Schirmer ehren.“ (Picc., V. 835) Gleichzeitig will er sich seine Machtspiele offenhalten und kontert den Vorwurf der Freunde, er habe den schwedischen Verhandlungspartner bloß ,zum besten“, in der Manier des arroganten Souveräns:

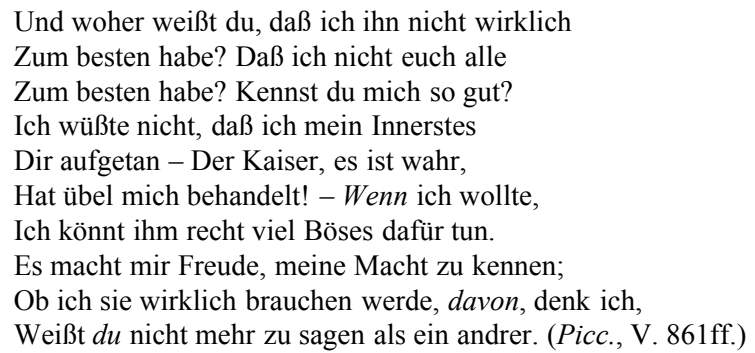

Das ist die Sprache des Machtpolitikers, der sich das Schauspiel der Undurchdringlichkeit leistet. Und der sein Ich selbst Freunden gegenüber bis zur Unkenntlichkeit vermummt. Aber es ist auch die Sprache des Abenteurers, der sich überschätzt und seinen Realitätssinn einzubüßen droht. Warum? Ein zusammenfassender Rückblick auf Wallensteins verschiedene Strategien und Projekte kann das verdeutlichen. Erkennbar wird damit zugleich das ganze Ausmaß seiner Selbsttäuschung.

\section{Wallensteins Selbsttäuschung}

Der Protagonist will

1. einer übergeordneten Reichsidee Realität verschaffen

2. seine Machtfülle durch die böhmische Königskrone steigern

3. die Feinde für seine Zwecke benutzen, gegeneinander ausspielen und hinters Licht führen

4. das Eintreffen einer günstigen Sternenkonstellation erwarten und dann alle drei Vorhaben in die Tat umsetzen

5. bis zu diesem Zeitpunkt auch die engsten Mitstreiter in Schach halten und auf sein Zögern verpflichten.

Fünf Vorhaben auf einmal - wie will Wallenstein sie koordinieren? Sie effektiv ineinandergreifen lassen? Ist er nicht überfordert durch die widerspruchsvolle Pluralität seiner politischen Ziele und mentalen 
Einstellungen? Der Ideenpolitiker, der ein übergeordnetes Allgemeininteresse anvisiert, der Träumer, der sich in seiner Machtfülle spiegelt, der auf Kriegsfuß mit seinem Kaiser lebende Machtpolitiker, der die eigene Machterweiterung verfolgt, der schillernde Taktierer und schauspielende Abenteurer, der die Feinde an sich binden und in die Irre führen will, der Metaphysiker und Pragmatiker schließlich, der eine kosmische Wesensschau betreibt und zugleich auf die stellare Empfehlung zur Tat wartet - wo wäre der Integrationspunkt dieser divergierenden Haltungen und Selbstrepräsentationen aufzufinden? Es scheint, als sei Wallensteins Identität in den Piccolomini aufgespalten in einander durchkreuzende Ichfragmente. Schiller hat seinen Helden in einer bestimmten Lebensphase mit einer diskordanten Vielstimmigkeit von politischen Mentalitäten ausgestattet, hat ihm ein heterogenes Selbst verliehen, wie es sich eher in moderner als in klassischer Literatur, eher in Werken des 20. als des 18. Jahrhunderts findet.

An keiner Stelle im Drama wird sich Wallenstein der widersprüchlichen Pluralität seiner politischen Ziele und mentalen Einstellungen, seiner heterogenen Identität in einer konkreten geschichtlichen Situation bewusst. Man könnte ebenso gut sagen, dass eine derartige Bewusstwerdung gerade durch seine Widerspruchsfülle verhindert wird. Wallensteins Bewusstseinsmangel ist das Einfallstor für seine Selbsttäuschung. Das Phantasma seiner königlichen Souveränität - „Es macht mir Freude, meine Macht zu kennen" - tritt in bewusstlosen Widerspruch zum zeitweiligen Eindruck eines baldigen Handlungszwangs. Als seine Gemahlin von einem Besuch des Wiener Hofs zurückkehrt und ihm von den dortigen Intrigen berichtet, die offenbar auf eine „Zweite Absetzung“ Wallensteins zielen, „schimpflicher“ als die erste beim Regensburger Fürstentag (Picc., V. 700), ist Wallenstein in seinen Grundfesten erschüttert: „O! sie zwingen mich, sie stoßen/ Gewaltsam, wider meinen Willen, mich hinein“ (Picc., V. 702f.). Der vor ihm auftauchende Handlungszwang ist das verbindliche Bündnis mit den Reichsfeinden, den Schweden oder Sachsen. Zwar kontert Wallenstein kurzfristig die ihm drohende Absetzung, indem er ein Schriftstück zirkulieren lässt, das seine Generale zu unbedingter Gefolgschaft ihm gegenüber verpflichtet (Picc. II, 6), doch darüber hinaus unternimmt er nichts, macht er von seiner Macht keinen weiteren Gebrauch, wird ihm ihre Infragestellung nicht bewusst. Dem Drängen seiner Berater, endlich entscheidende Schritte zu unternehmen, erteilt er einen abschlägigen Bescheid: „Die Zeit ist noch nicht da" (Picc., V 958) und verweist in episch breiter Rede auf die „Sternenwelt“ und „rechte Sternenstunde“ (Picc., V. 966-1002). Allem Anschein nach möchte er die desintegrativen Handlungsimpulse und interessen, die sein Bewusstsein nicht selbstkritisch zu überblicken vermag, in seiner Astrologie als übergeordneter Instanz vereinigen, ideell und praktisch. Ideell, insofern sie ihm als metaphysische „Geisterleiter“ von den „Tiefen der Natur“ hinauf zu den „himmlischen Gewalten“ dient (Picc., V. 978ff.), praktisch, insofern sie ihm die „Saatzeit" für „,des Menschen Tun“ angeben soll (Picc., V. 990ff.). Schillers dramatische Ironie ist an dieser Stelle 
zwingend. Octavio, den Wallenstein für seinen verlässlichsten Weggefährten hält, trägt schon „den kaiserlichen Brief" bei sich, der die Entmachtung und Ächtung Wallensteins anordnet (Picc., V, 1). Obgleich die Freunde ihren Feldherrn vor Octavio warnen, verteidigt Wallenstein ihn astrologisch: „Wir sind geboren unter gleichen Sternen -“(Picc. V. 889). Mitten in seiner Sternenschau ist Wallenstein blind für die Realität - und verharrt in dieser Blindheit wie unter einem Zwang, dem traumatischen Zwang eines Gedemütigten, der sich an eine private Sinngebung klammern muss. So belügt er sich selbst. Auch als die verheißungsvolle Sternenkonstellation endlich eintrifft, jedoch mit der Gefangennahme Sesins, des Unterhändlers Wallensteins, kollidiert, zweifelt Wallenstein nicht einen Augenblick an der Richtigkeit der astrologischen Aussagekraft. Und als wenig später die engsten Ratgeber ihn auf Octavios heimliche Machenschaften hinweisen und ihn unter Aufbietung aller Rednerkünste als seinen Gegner entblößen, ignoriert Wallenstein ihre Gründe mit der Behauptung, seine Astrologie entspringe der „,tiefsten Wissenschaft“, und diese bürge fraglos für Octavio: „Lügt $e r$, dann ist die ganze Sternkunst Lüge.“" (Tod, V. 891f.)

Lüge ist sie an dieser Stelle in der Tat. Sie mag Wallensteins dramatische Verunsicherung dämpfen, doch als praktische Anleitung zum Handeln ist sie untauglich. Widerrufen wird sie nicht nur durch die Gefangennahme Sesins und davor schon durch Octavio, den Geheimagenten des Kaisers, widerrufen wird sie auch durch die Strategie der Reichsfeinde. Ihnen dünkte sich Wallenstein stets überlegen ${ }^{7}$, und als der „glückselige Aspekt" der Gestirne endlich eintrifft (Tod, V. 9), darf er hoffen, sie seinen Interessen nachdrücklich zu unterwerfen. Narzisstisch sich spiegelnd in seinem Traum von der eigenen Größe, befangen in der Illusion, die Reichsgegner seien seiner Strategie nicht gewachsen, hatte er sie hingehalten und auf die verheißungsvolle „Sternenstunde“ gewartet. In der Zwischenzeit hatten die Gegner das Katz-und-Maus-Spiel des politischen Traumwandlers und Sterndeuters mit einem ausgeklügelten Schachzug hintertrieben; als Wallenstein aufgrund der Gefangennahme Sesins mit den Schweden eine Gegenstrategie zu den Intrigen des Wiener Hofs entwickeln will, hält das schwedische Heer einen Teil Prags, der Hauptstadt Böhmens, bereits besetzt; auch die strategisch wichtige Stadt Eger soll sich dem Reichsfeind „öffnen“ (Tod, V. 390ff.). Die böhmische Königskrone, von der Wallenstein inständig träumte, kann der Schwede ihm nach Belieben vorenthalten. Abermals hat die konkrete Sternenkonstellation ihn getäuscht; als sie ihm endlich den Zeitpunkt des Handelns verheißt, ist ihm das Gesetz des Handelns schon abhanden gekommen. Der narzisstische Träumer und Zögerer hat die Realität so lange verkannt und sich selber so lange in Illusionen gewiegt, bis aus dem Traum von der eigenen Macht der Alptraum von der Teilung der Macht mit dem Reichsfeind geworden ist.

\footnotetext{
${ }^{7} \mathrm{Zu}$ den Schwächen des Wallensteinschen Sternenglaubens vgl. die scharfsinnige Kritik bei Peter-André Alt (Anm. 2), S. 445ff.
} 


\section{Monologische Wahrheitssuche}

Erst jetzt, in dieser Zwangslage, stellt sich Wallenstein seiner Selbsttäuschung und versucht ihr auf den Grund zu gehen. Er tut dies bezeichnenderweise in der Redeform des Monologs. Monologe zeichnen etliche Helden Schillers aus - von den Brüdern Moor in den Räubern über Fiesco zu Maria Stuart, zur Jungfrau von Orleans und zu Wilhelm Tell zieht sich ein respektabler Bogen von Selbstbefragungen, in denen die Hauptfigur sich zur Rechenschaft zieht. Das pietistische Erbe Schillers meldet sich da zu Wort und verschafft der Gewissensstimme Gehör - der Wahrheit zu Ehren, die das Gespinst der Selbsttäuschung zerreißen soll. Es sind krisenhafte Grenzsituationen, wie sie im 20. Jahrhundert von der Existenzphilosophie eines Jaspers, eines Camus und eines Sartre offengelegt wurden. Das für sich selbst verantwortliche Individuum steht in der Freiheit der Entscheidung über Wahrheit oder Selbsttäuschung, über Moral oder Unmoral. Schillers Hauptfiguren zählen zur Avantgarde einer selbstkritischen, mit der Gewissensstimme ringenden Moderne. Wallenstein zögert denn auch nicht, seine „Versuchung“ zu bekennen: seinen „Traum“ von einer gesteigerten Machtfülle und seine „königliche Hoffnung“ auf eine Krone weitausgreifende Wünsche, die er bedenkenlos dem freien Wort anvertraute; verführt von der Leidenschaft der Affekte und vom „Überfluß des Herzens“, öffnete er die Schleusen der Sprache und erging sich in rebellischen Gedankenspielen: „Kühn war das Wort, weil es die Tat nicht war.“ (Tod, V. 170) Wallenstein geht mit sich selbst als einem Macht-Träumer und Macht-Spieler offen ins Gericht, er gesteht die Gedankenspiele, die offensichtlich auf Landesverrat gemünzt waren, doch er beharrt auf der Unterscheidung zwischen Spiel und Ernst, zwischen „unverführtem Willen“ und ausgeführter Tat: „Beim großen Gott des Himmels! Es war nicht/ Mein Ernst, beschloßne Sache war es nie./ In dem Gedanken bloß gefiel ich mir". (Tod, V. 146ff.) Diese feine Unterscheidung hält nicht ganz der Überprüfung stand. Lag da in Wahrheit nicht eine Grenzverwischung vor? Ist ein mit Dokumenten versorgter Unterhändler wie Sesin denn nicht mehr als nur ein Gedankenspiel, das da unverbindlich-folgenlos durch den politischen Raum dahintreibt? Ist er nicht die Fleischwerdung dieses Spiels, seine Überführung in die Realität? Wohl erkennt Wallenstein klarsichtig, dass Reden, die er „planlos“ und im Affekt führte, beispielsweise vor Sesin, nun vom Wiener Hof und dessen Anhängern „planvoll“ zu einem „künstlichen Gewebe“ verknüpft werden, zu einer Anklage, gegen die er „verstummen muß“ (Tod, V. $171 \mathrm{ff}$.). Aber er verschließt sich der Einsicht, dass sein Spiel schon Züge des Ernsts hatte und sein Gedankenexperiment in die Realität übergriff. ${ }^{8}$ Offenbar benötigt er zu seiner Selbstverteidigung ein Zeugnis moralischer Integrität: „Der Unschuld,/ Des unverführten Willens mir bewußt“, beteuert

\footnotetext{
${ }^{8}$ Treffend formuliert Safranski (Anm. 6): „Wallenstein hat mit dem Verrat gespielt, halb noch in seinem Herzen, halb schon in der Wirklichkeit." (S. 460).
} 
er, habe er Bedenkliches und Verräterisches geäußert: „Gab ich der Laune Raum, der Leidenschaft -“ (Tod, V. 167ff.). Wenn jedoch derartige Äußerungen einem Mittelsmann und Boten anvertraut werden, der zwischen Wallenstein, seinen Ratgebern und dem Reichsfeind hin und her pendelt, so werden die Grenzen zwischen „Wort" und „Tat", mündlichem Spiel und faktischem Tun, sprachlichem Probehandeln und realer Handlung durchlässig. Auch in seinem Monolog, der die Wahrheit über das eigene Selbst erkunden soll, streift Wallenstein den Schleier der Selbsttäuschung nicht vollkommen ab.

Es fällt auf, dass Wallenstein in dieser Selbstbefragung nur einen Teil seines jüngsten Soldatenlebens in Betracht zieht, namentlich seine angebliche Verantwortung für den Reichsverrat, den man ihm zur Last legen wird. Er wirft darüber hinaus noch einen kritischen Blick auf die Mentalität seiner Zeitgenossen, die er als Parteigänger des „ewig Gestrigen“ und der "Gewohnheit" geißelt, dem Herkommen verschworen, der herrschenden „Macht“, der „sicher thronenden“, gedankenlos treu, abgeneigt jeder Neuerung und Neuordnung der bestehenden Verhältnisse, die er, Wallenstein, offenbar anstrebt (Tod, V. 192ff.). Was er unter seinem Angriff auf die überkommene „Macht“ versteht, bleibt ungesagt. Eine neue Friedensordnung zugunsten des Reichs und zu seinen eigenen Gunsten? Ebenso wenig bringt er seinen Sternenglauben mit seinen vielfältigen Implikationen zur Sprache. Die Widerspruchsfülle seiner politischen Interessen und seiner Persönlichkeit insgesamt bleibt verborgen. Sie ist zu komplex, als dass sie sich in einem einzigen, monologischen Bewusstseinsakt erfassen ließe. Statt dessen kreist Wallensteins Selbstdurchleuchtung, die Schiller mit einem glänzenden rhetorischen Aufwand an Metaphern, Antithesen und variantenreichen Wiederholungen ausgestattet hat, zentral um die Frage, ob er den Reichsverrat verbindlich geplant hat und ihn nun auch, notgedrungen, begehen müsse, um ein Gegengewicht gegen die ihm drohende Absetzung zu schaffen. Den schwedischen Oberst Gustav Wrangel erwartend, der mit ihm ein definitives Bündnis erörtern will, formuliert Wallenstein, „,en Blick nachdenkend auf die Türe geheftet", die entscheidungsschweren Sätze:

Noch ist sie rein - noch! Das Verbrechen kam

Nicht über diese Schwelle noch - So schmal ist

Die Grenze, die zwei Lebenspfade scheidet! (Tod, V 220ff.)

\section{Die Wahrheit auf dem Prüfstand. Politik contra Moral}

Wenn Wallenstein am Ende seines Monologs sich vor den Widerstreit zweier „Lebenspfade“ gestellt sieht, so rücken damit auch die Wahrheit und die Moral seiner künftigen Existenz in sein Blickfeld. Kann er, der sich wiederholt als Maskenspieler inszeniert hat, aber auch der Selbsttäuschung erlegen ist, zu einer Wahrheit finden, die den Ansprüchen humanitärer Moral genügt? Oder muss er sich dem Gebot einer amoralischen Politik beugen und 
ein „Verbrechen“ auf sich laden? Und ist dieses Verbrechen nicht mit einer eigentümlichen, auf Wallenstein persönlich zugeschnittenen ,Wahrheit' verschränkt, gleichsam seinem Lebensgesetz? Schillers dramatischer Vorgang besitzt eine hohe moralische und existentielle Spannung.

Das „Verbrechen“, das vor Wallensteins Augen heraufzieht, wäre ein Krieg, den er gemeinsam mit den Schweden und mit Teilen des kaiserlichen Heers - den ihm ergebenen Teilen - gegen den Kaiser und damit gegen das Reich führen müsste. Es wäre ein Bürgerkrieg, den er im Reichsheer selbst entfesseln würde. Davor schreckt er zurück. Es wäre Hochverrat. Es wäre das militärische „Verbrechen“ schlechthin, die radikale Verwerfung des moralischen Gesetzes. Im Namen der Moral hält Wallenstein Gericht über die bevorstehende Untat, stellt er sich selbst elementar in Frage, malt er sich den Fluch aus, den er sich damit einhandeln, die allgemeine Ächtung, die er auf sich ziehen würde:

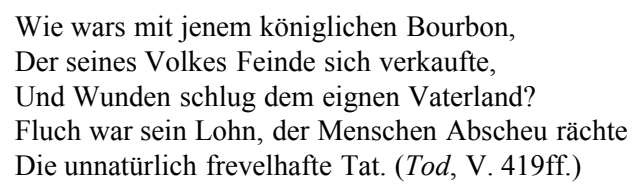

Schiller hat die Selbstdarstellung der Moral als Stimme des Gewissens wiederholt erprobt, sie zieht sich leitmotivisch durch sein Werk. Wallenstein wird nachgerade zum Organ der Moral, wenn er seine ihn bedrängenden, ihn zum Verrat drängenden Freunde in Schach hält, wenn er ihnen eine Lektion erteilt im Namen der Treue: der Treue, die für die Integrität zwischenmenschlicher Beziehungen bürgt, der Loyalität, ohne die keine Gemeinschaft gedeihen kann:

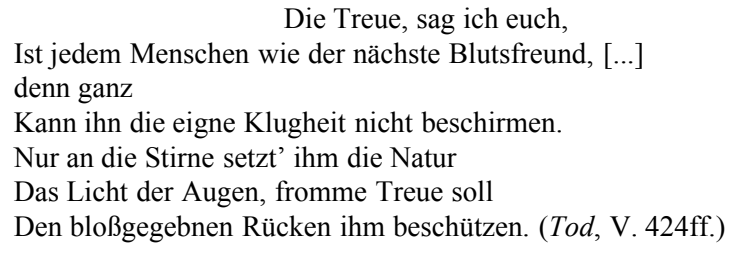

Wallenstein formuliert eine Grundregel jeder Zivilisation, eine moralische, überzeitliche Wahrheit: eine menschliche Gemeinschaft kann sich vielleicht nicht schützen gegen die Angriffe eines äußeren Feinds, wohl aber muss sie sich schützen gegen Überfälle, Totschlag und Mord in ihren eigenen Reihen. Sonst ist zivilisiertes Leben nicht möglich. Die Sicherheit jeder persönlichen Existenz und des Alltagslebens wäre unter der permanenten Gefahr heimtückischer Anschläge aufgehoben. Einen derartigen Anschlag, geführt gegen sein eigenes Heer, gegen Kaiser und Reich, fürchtet Wallenstein zu begehen. Daher eröffnet er, im Namen der moralischen Wahrheit, den Kampf gegen sich selbst und das drohende militärische Unternehmen. Er sucht nach 
Alternativen, verzweifelt, will die Freveltat und die mit ihr gesetzte Schuld vermeiden, unter allen Umständen:

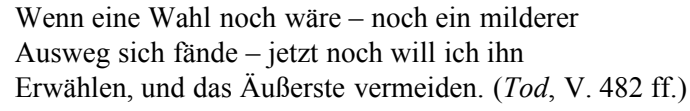

Wallenstein zögert - doch diesmal ist es kein berechnetes, unter dem Einfluss der Sterne stehendes Zögern, sondern ein von Moral und politischer Norm bestimmtes. In dieser Situation gewinnt er eine menschliche Größe wie nie zuvor. Paradigmatisch lebt er vor, was jeder Politiker von Rang und von Verantwortungsbewusstsein nachvollziehen müsste: die Überprüfung schwerwiegender Entscheidungen unter dem Gesichtswinkel des moralischen Gesetzes. Dieses Gesetz existiert, es hat die Zeit der deutschen Klassik überdauert und ist in aktueller Gestalt niedergelegt in ethischen Instanzen wie den Menschenrechten und dem Völkerrecht, mögen auch einzelne Großmächte bzw. deren Machtpolitiker solche Instanzen geringschätzen.

\section{Die Attraktivität der Halbwahrheit}

Es macht die Tragik Wallensteins aus, dass er die von ihm selbst formulierte normative Wahrheit nicht respektieren kann. Auf der Suche nach Alternativen zum Reichsverrat trifft er auf die Gräfin Terzky - und diese Frau präsentiert ihm den einzig möglichen Ausweg: den Rückzug in den Ruhestand als harmloser Schlossherr und ruhmloser Mäßiggänger. Sie präsentiert ihn mit so spitzer Ironie, dass Wallenstein sich mit einem Schlag bewusst wird: ein Privatleben, das abseits der politischen Öffentlichkeit verläuft und allem politischen Handeln fern steht, würde den Kern seiner Identität angreifen. Und Wallenstein besitzt, unbeschadet seiner Vielheit an wechselnden Ich-Gestalten, einen Identitätskern. Er fasst ihn in einen einzigen Satz: „Wenn ich nicht wirke mehr, bin ich vernichtet“ (Tod, V. 528). Dem moralischen Gesetz stellt er das Gesetz seiner Existenz gegenüber, jenes "Gesetz“, wonach er „,angetreten“, um mit Goethe zu sprechen", und dieses Gesetz lautet: Wirken im Sinne von wirkungsvoll handeln gemäß dem ihm eingeborenen Genius. Kann, muss einer seinen Genius verleugnen, sein Lebensgesetz auslöschen um der moralischen Wahrheit willen? Wallenstein jedenfalls vermag gerade das nicht:

Nicht Opfer, nicht Gefahren will ich scheun,

Den letzten Schritt, den äußersten, zu meiden;

Doch eh ich sinke in die Nichtigkeit,

\footnotetext{
${ }^{9}$ In den „Urworten. Orphisch“ heißt es: „Bist alsobald und fort und fort gediehen/ Nach dem Gesetz, wonach du angetreten." (GOETHE, Gedichte, herausgegeben und kommentiert von Erich TRUNZ [Sonderausgabe von Bd. 1 der „Hamburger Ausgabe“], München, C. H. Beck, 1974, S. 359).
} 
So klein aufhöre, der so groß begonnen, [...]

Eh spreche Welt und Nachwelt meinen Namen [...]

Mit Abscheu aus [...].(Tod, V. 528ff.)

Wallenstein ist nach innerem Kampf entschlossen, die Erfüllung seines individuellen Gesetzes um jeden Preis zu erreichen. Schiller zeigt an seinem Protagonisten kritisch die Gefahr auf, die in der Individualitätsidee verborgen ist: die Selbstüberhebung ohne Rücksicht auf die „Wohlfahrt aller“, auf „des Ganzen Heil“, wie es Wallenstein einmal vorschwebte. ${ }^{10}$ Die moralischen Skrupel, die ihn an diesem Wendepunkt seines Lebens heimsuchen, zerstreut die Gräfin Terzky. Diese Dame besitzt eine bemerkenswerte Intelligenz und verfügt über die Kunst einer unwiderstehlichen Rhetorik. Gescheiter und wortgewandter als die Männer in Wallensteins Umfeld gehört sie in die Reihe jener weiblichen Gestalten Schillers, welche die traditionelle Frauenrolle in seiner Zeit sprengen. Die Gräfin verweist Wallenstein zunächst auf das Naturgesetz der Notwehr, die jedem bedrängten und bedrohten Subjekt zustehe, sie erinnert ihn dann an die ihm vom Kaiser zugefügte „Kränkung“ in Regensburg (Tod, V. 554), ruft ihm ins Gedächtnis, dass nicht etwa das Vertrauen, sondern allein die „herbe Not" des Kaisers ihm sein gegenwärtiges „Amt" verschafft habe (Tod, V. 570) und dass er dem Kaiser menschlich zu nichts verpflichtet sei; sie überzeugt ihn schließlich davon, dass er immer schon als Feldherr „der Stärke fürchterliches Recht“ ausgeübt habe (Tod, V. 607), vor allem auch in Kaisers Diensten, und sie triumphiert mit der glänzend formulierten Pointe:

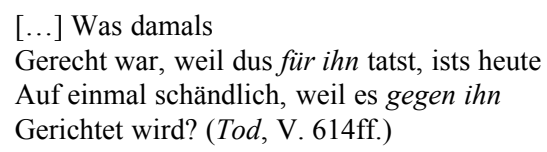

Geflissentlich verwischt die Gräfin den Unterschied zwischen „damals“ und „heute": Damals hat Wallenstein zwar wie eine Kriegsfurie, aber im Namen des Kaisers, das Land durchzogen, heute soll er als Hochverräter gegen Kaiser und Reich zu Felde ziehen. Indem die Gräfin diese Differenz einebnet und das moralische Gesetz zum Schweigen bringt, kann sie noch eine Trumpfkarte ziehen. Sie redet dem Feldherrn die Kontinuität seines „Charakters“ seit damals bis heute ein und verpflichtet ihn darauf:

Denn Recht hat jeder eigene Charakter,

Der übereinstimmt mit sich selbst, es gibt

Kein andres Unrecht, als den Widerspruch. (Tod, V. 600ff.)

\footnotetext{
${ }^{10}$ In seinem bedeutenden Schiller-Buch verkennt Müller-Seidel (Anm. 4) diese Entwicklungsphase Wallensteins, wenn er seine Friedensidee als sein zentrales politisches Projekt bezeichnet; mit dem Entschluss zum Reichsverrat setzt Wallenstein an dieser Stelle nachgerade einen Bürgerkrieg in Gang.
} 
Die Idee des mit sich selbst übereinstimmenden Charakters ist für sich betrachtet durchaus plausibel. Sie wird im Kontext der dramatischen Situation jedoch zu einer destruktiven Halbwahrheit. Die Gräfin vereinfacht damit das widerspruchsvolle Ich Wallensteins radikal - sie stellt sein Lebensgesetz, die politische Tatkraft, wieder her, befreit ihn jedoch von moralischen Skrupeln und vom Ethos einer überpersönlichen politischen Zielsetzung. Doch gerade diese Halbwahrheit besitzt eine hohe Suggestionskraft. Eine von Skrupeln freie Identität, wie die Gräfin sie fingiert, ist ein verführerisches Wunschbild für jemanden, der eben noch mit den Ansprüchen politischer „Treue“ und den Anfechtungen der Moral zu ringen hatte, ja, von der Moral gleichsam mit sich selbst entzweit wurde. An ihre Stelle und an die Stelle der Selbstentzweiung zaubert die Gräfin dem Zögernden und Zweifelnden die amoralische Übereinstimmung mit sich selbst vor Augen und gewinnt ihn für das Bündnis mit dem Reichsfeind. So macht denn Wallenstein unter dem Zwang seines individuellen Lebensgesetzes und der geschichtlichen Verhältnisse erneut eine Wandlung durch: die vom komplexen und widerspruchsvollen Ich zum einschichtigen Charakter, der sowohl die moralische Wahrheit wie sein übergeordnetes politisches Ethos preisgibt. Solche zugleich psychologisch und geschichtlich motivierten Veränderungen einer Person gehören zu den modernen Zügen der Figurengestaltung Schillers. Man kann als Leser der Gräfin wohl kaum einen leisen ästhetischen Applaus versagen. Obgleich sie bar jeder Moral argumentiert, ja, der Unmoral die Zunge leiht, macht sie das mit Geist und mit psychologischem Scharfsinn. Gegen die Widerspruchsfülle Wallensteins spielt sie mit glanzvoller Überredungskunst die Halbwahrheit eines vereinfachten, mit sich identischen Selbst aus. Das individuelle Lebensgesetz, dem Wallenstein folgt, und die Wahrheit der Moral bzw. einer überpersönlichen Idee, der er folgen sollte, weisen unversöhnlich auseinander. Die Antinomie, in die Schiller seinen Helden verstrickt, ist wahrhaft desperat.

Im Zuge seiner Selbsttäuschung hat Wallenstein, wie er in seinem Monolog klarsichtig erkannte, ,,mit eignem Netz verderblich mich umstrickt" (Tod, V. 178), doch ebendiese Verstrickung hat bereits Octavio besorgt. Ihm, dem langjährigen „Waffenbruder“ (Picc., V. 357), hatte er bedingungslos vertraut, während Octavio den arglosen Freund gleichzeitig zu Fall brachte. Verstellung, Missdeutung, Halbwahrheiten, Denunziation - alle Halbschwestern der Lüge hat Octavio im Dienst des Kaisers virtuos eingesetzt. ${ }^{11} \mathrm{Zu}$ spät durchschaut Wallenstein den kaltblütigen Gegenspieler:

Am Sternenhimmel suchten meine Augen,

Im weiten Weltenraum den Feind, den ich

Im Herzen meines Herzens eingeschlossen. (Tod, V. 2105ff.)

Wallenstein formuliert damit explizit seine astrologische Selbsttäuschung, die Octavios Verrat erst ermöglichte. In ihm zeichnet Schiller den

\footnotetext{
${ }^{11}$ Zur Gestalt Octavios vgl. meine Ausführungen im Aufsatz von 2006 (Anm. 1).
} 
skrupellosen Politiker par excellence, der frei von menschlichen und moralischen Bedenken, weitsichtig und einfallsreich agiert. Eben damit stürzt er seinen Sohn Max in Verzweiflung, wird er mitschuldig an seinem Tod, gemeinsam mit Wallenstein, dem zweiten „Vater“ des jungen Offiziers.

\section{Reduzierte Identität und die Wahrheit zum Tode. Wallenstein und Max Piccolomini}

Im Namen der Wahrheit stellt Max Piccolomini den Feldherrn, sein väterliches Vorbild, zur Rede. Unter Berufung auf ethische Normen versucht er Wallenstein vom Hochverrat im letzten Augenblick fernzuhalten. Der junge General kommt Wallenstein großzügig, erstaunlich großzügig entgegen, würde er doch eine „offene Empörung“ seines Feldherrn gegen den Kaiser noch tolerieren (Tod, V. 770ff.). Der Reichsverrat jedoch, das Bündnis mit dem Reichsfeind, den Schweden, ist für ihn das schlechthin Unverzeihliche, der Affront gegen das moralische Gesetz, beispiellos, unter der Würde jeden Politikers. Wallenstein hält der Moral seines jugendlichen Anklägers nüchtern die unvermeidliche Unmoral des Realpolitikers entgegen. Ihr will er zuletzt auch den jungen Freund zynisch unterwerfen:

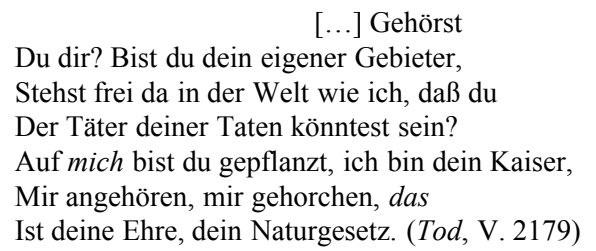

Es ist die Rhetorik des moralfreien Machtpolitikers, die Wallenstein anstimmt. Die neue, vereinfachte und verkürzte Identität, die er sich angeeignet hat, verführt ihn dazu, einer anderen Person die eigene Identität streitig zu machen und sie zum Exekutivorgan seiner Untat zu bestimmen. Die Existenz des jungen Piccolomini würde damit der Unwahrheit preisgegeben - der vollständigen Selbstentfremdung. Wallensteins täuschende Rede zielt auf den Missbrauch einer langjährigen Freundschaft für eine politische Katastrophe. Der junge Piccolomini widersteht jedoch der monströsen Egozentrik des Freundes und zweiten Vaters. Die „Stimme der Wahrheit" (Tod, V. 2295f.) gebietet ihm die Trennung vom Reichsverräter Wallenstein. So wird er „mündig“, wie er von sich selber sagt (Tod, V. 711). Er wird es unter Schmerzen, weil er dem Widerstand leisten muß, der bis jetzt „wie der feste Stern des Pols“ ihm ,als die Lebensregel vorgeschienen“ (Tod, V. 734f.). Piccolomini muss sich von einem wesentlichen Teil seiner IchIdentität emanzipieren, jenem, der sich um die Person Wallensteins kristallisiert hat. Schiller durchdringt die Emanzipation des jungen Offiziers mit scharfsichtiger Psychologie. Zwar „befreit“ sich die Seele Piccolominis 
von ihrer Vatergestalt und ihrem Lebenszentrum, dennoch sind seine „Sinne“ noch in den „Banden“ Wallensteins, dessen „Angesicht" ihm bis zur Stunde „eines Gottes Antlitz“ war (Tod, V. 739ff.). Dass die sinnliche Ausstrahlung eines lange geliebten Menschen die mentale und psychische Entscheidung gegen ihn überdauern kann, zeigt die Abgründigkeit der Vater-Ablösung Piccolominis an. Die Sinne können ihre eigene Sprache reden - im Widerstreit mit der Erkenntnis der Wahrheit. Schiller greift noch einmal die Vater-Sohn-Problematik seiner Jugenddramen von den Räubern über Kabale und Liebe bis zu Don Carlos auf. Greift sie auf und verankert sie noch einmal im Konfliktfeld von Politik und Moral. Verankert und verdoppelt sie zugleich, indem er der Vatergestalt Wallensteins die noch problematischere des Octavio zugesellt. Mit dem Pathos einer unverbrüchlichen Moral und seiner aussichtslosen Liebe zu Wallensteins Tochter Thekla klagt Max „der Väter Doppelschuld und Freveltat“ an, die „uns gräßlich wie ein Schlangenpaar umwinden“ (Tod, V. 2138f.). Er sieht keinen Ausweg aus dieser Umklammerung, weder einen als politisch Handelnder, noch einen als Liebender. Bestürzt über Octavio, seinen leiblichen Vater, der an Wallenstein, dem Vater seines Herzens, zum Verräter geworden ist, verzweifelt über den Reichsverräter Wallenstein, gegen den er gleichwohl nicht zu Felde ziehen möchte, sucht er den Tod in der Schlacht. Aber vor lauter Treue zum eigenen moralischen Selbst missbraucht auch er seine Macht, und setzt in obsessiver Ichbezogenheit sein ganzes Heer dem Untergang aus. In seiner bedingungslosen Negation der väterlichen Welt muss er dieser gleichwohl seinen Tribut entrichten. Schillers dialektische Gestaltung verklammert subtil die drei Hautfiguren miteinander.

Die Väter jedoch verrechnen sich gründlich. Mit der Nachricht von seiner Erhebung in den Fürstenstand erhält Octavio zugleich die vom Tod seines Sohns. Der Fürst Piccolomini, der keinen Erben mehr besitzt, hat seine neue Würde teuer erkauft. Und Wallenstein? Seine Todesklage gehört zu den ergreifendsten in der deutschen Literatur. Der Real- und Machtpolitiker erkennt durch den Untergang seines jugendlichen Weggefährten die Armut seines neuen, flacheren Selbst: erkennt, was er in Max Piccolomini verloren hat, verloren auf immer: das Ideal, die Poesie, das Feuer des Empfindens und Liebens, die eigenen Jugendträume, die Sehnsucht, die hinausweist über die nüchterne Pragmatik des Kampfes, des Erfolgs, des Alltags. Mit dem Tod Piccolominis hat Wallenstein all das eingebüßt, was das Leben lebenswert macht. Indem er über diesen Verlust so beredt Klage führt, rührt er noch einmal an die menschliche Größe, die er im politischen Handeln verdrängen musste:

Verschmerzen werd ich diesen Schlag, das weiß ich,

Denn was verschmerzte nicht der Mensch! Vom Höchsten

Wie vom Gemeinsten lernt er sich entwöhnen,

Denn ihn besiegen die gewaltgen Stunden.

Doch fühl ichs wohl, was ich in ihm verlor.

Die Blume ist hinweg aus meinem Leben, 


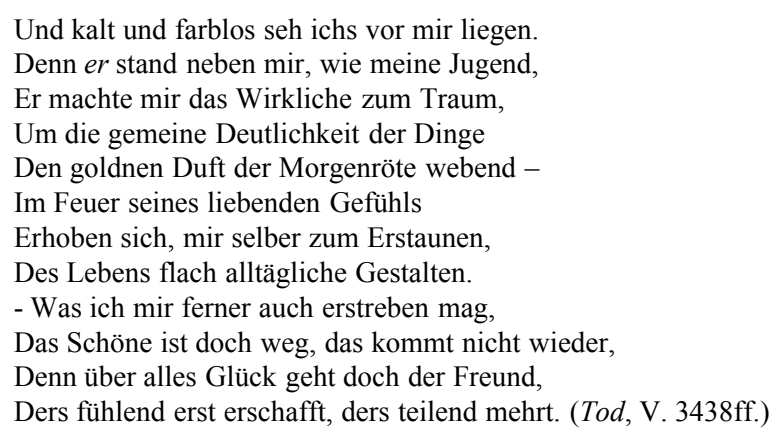

Es ist kein Zufall, wenn diesem Bekenntnis eine Verwechslung vorausgeht, die das sinnvolle Ineinanderspiel zweier Fixsterne im Leben Wallensteins bedeutet. In die Betrachtung des Nachthimmels versunken, hielt er vergeblich nach Jupiter Ausschau -

Es ist der Stern, der meinem Leben strahlt,

Und wunderbar oft stärkte mich sein Anblick. (Tod, V. 3417)

- worauf er, „in eine tiefe Zerstreuung“ fallend, unbewusst Jupiter mit der Gestalt des toten Piccolomini assoziierte. Die in dieser Gestalt verkörperte Poesie und Idealität, Ideenwelt und seelische Intensität sind Teil der Sehnsuchtsbilder, die Wallenstein in seinem Idealstern symbolisiert sah. ${ }^{12}$ Der zuletzt im Banne Saturns, des ernüchternden Realitätsprinzips, agierende Feldherr bleibt trauernd auf das Traumbild bezogen, das er im „Notzwang der Begebenheiten" (Picc., V. 1367) verraten musste.

Wallensteins Trauer angesichts des Untergangs des „Schönen“ berührt sich mit der Klage Theklas über den Tod des Geliebten: „- Das ist das Los des Schönen auf der Erde.“ (Tod, V. 3180) - Das Schöne als Person hat in der vom Krieg heimgesuchten Geschichte kein Existenzrecht. Aber es überlebt den Tod der Person als Idee. Die von Max Piccolomini anfangs entworfene Friedensvision enthält das Attribut „schön“ als Hinweis auf jene Utopie, die es - alternativ zur Geschichte des Dreißigjährigen Kriegs und aller nachfolgenden Kriege - herzustellen gilt: „O schöner Tag, wenn endlich der Soldat/ Ins Leben einkehrt, in die Menschlichkeit" (Picc., V. 534f.). Die Wahrheit dieser Utopie ist dem Wallenstein-Drama als Geheimschrift eingezeichnet - in stummer Opposition zur vielzitierten Rede von der nihilistischen und „fatalistischen Stimmung“, die Hegel zur eigentlichen Botschaft dieser Tragödie erklärte. ${ }^{13}$

\footnotetext{
${ }^{12}$ Vgl. dazu Verf.: Idyllik und Dramatik im Werk Friedrich Schillers. Zum geschichtlichen Ort seiner klassischen Dramen, Stuttgart, W. Kohlhammer, 1971 (3. Kap.).

${ }^{13}$ Zit. nach ALT, Schiller, S. 461. Mit Recht weist dagegen Müller-Seidel (Anm. 4) auf den „,Trost“ des Erkennens“ am Ende der Tragödie hin (S. 143).
} 